\title{
ON COMPLETE MANIFOLDS WITH NONNEGATIVE RICCI CURVATURE
}

\author{
UWE ABRESCH AND DETLEF GROMOLL
}

Complete open Riemannian manifolds $\left(M^{n}, g\right)$ with nonnegative sectional curvature are well understood. The basic results are Toponogov's Splitting Theorem and the Soul Theorem [CG1]. The Splitting Theorem has been extended to manifolds of nonnegative Ricci curvature [CG2]. On the other hand, the Soul Theorem does not extend even topologically, according to recent examples in [GM2]. A different method to construct manifolds which carry a metric with Ric $>0$, but no metric with nonnegative sectional curvature, has been given by L. Bérard Bergery [BB]. This leads to the question (cf. also [Y1]): Is there any finiteness result for complete Riemannian manifolds with $\mathrm{Ric} \geq 0$ ? The answer is certainly affirmative in the low-dimensional special cases $n=2$, where all notions of curvature coincide, and $n=3$, where nonnegative Ricci curvature has been studied by means of stabie minimal surfaces [MSY, SY]. On the other hand, J. P. Sha and D. G. Yang [ShY] have constructed complete manifolds with strictly positive Ricci curvature in higher dimensions. For example they can choose the underlying space to be $\mathbf{R}^{4} \times S^{3}$ with infinitely many copies of $S^{3} \times \mathbf{C P}^{2}$ attached to it by surgery; cf. also [ShY1]. It is therefore clear that any finiteness result for arbitrary dimensions requires additional assumptions.

The purpose of this paper is to establish the following main result.

Theorem A. Let $M^{n}$ be a complete open Riemannian manifold with Ric $\geq 0$. Suppose that $M^{n}$ has diameter growth of order $a\left(r^{1 / n}\right)$. Then $M^{n}$ is homotopy equivalent to the interior of a compact manifold with boundary, provided the sectional curvature is bounded away from $-\infty$.

The notion of diameter growth requires a precise definition. Roughly speaking, we would like to measure the diameters of the "essential components" of the distance spheres $S\left(p_{0}, r\right)$ w.r.t. the intrinsic metric in $M^{n} \backslash B\left(p_{0}, \zeta \cdot r\right)$, where $\frac{1}{2}<\zeta<1$ is a fixed number. Given any open set $\Omega \subset M^{n}$, not necessarily connected, we shall write $\operatorname{diam}(\Sigma, \Omega)$ for the diameter of any connected subset $\Sigma \subset \Omega$ measured w.r.t. the intrinsic distance function of the open submanifold

Received by the editors June 20,1989; part of this paper has been presented at the 838th meeting of the American Mathematical Society, University of California, Los Angeles, November 15, 1987.

1980 Mathematics Subject Classification (1985 Revision). Primary 53C20, 31C12.

The first author was partially supported by D.F.G. and the second author by N.S.F. Grants DMS 8405956 and DMS 8705721. 
$\Omega$. Let $C\left(p_{0}, r\right)$ denote the union of the unbounded connected components of $M^{n} \backslash \overline{B\left(p_{0}, r\right)}$. We set

$$
\operatorname{diam}\left(p_{0} ; r\right):=\sup \operatorname{diam}\left(\Sigma_{k}, C\left(p_{0}, \zeta r\right)\right),
$$

where the supremum is taken over all components $\Sigma_{k}$ of $\partial C\left(p_{0}, r\right)$.

Definition. Let $f: \mathbf{R}^{+} \rightarrow \mathbf{R}^{+}$be a monotonic function. A Riemannian manifold $M^{n}$ with base point $p_{0}$ is said to have diameter growth of order $o(f)$ (resp. $\mathscr{O}(f)$ ), if and only if $f(r)^{-1} \cdot \operatorname{diam}\left(p_{0} ; r\right)$ converges to zero as $r \rightarrow \infty$ (resp. remains bounded).

This definition will be discussed further in $\S 1$. Here we would just like to point out that the details have been arranged in such a way that the diameter growth condition in Theorem A is as little a restriction as possible. The reason for taking the supremum in formula (0.1) rather than a sum or any other norm becomes even more clear when we present our result in a slightly more general context. Quite in contrast to the Splitting Theorem in [CG2], Theorem A extends to manifolds with asymptotically nonnegative Ricci curvature, thus going beyond a rigidity result.

Theorem B. Let $M^{n}$ be a complete open Riemannian manifold with base point $p_{0}$, and let $r_{0}(q)=d\left(p_{0}, q\right)$ for all $q \in M^{n}$. Suppose that

(0) there is a nonincreasing function $\lambda:[0, \infty) \rightarrow[0, \infty)$ such that $C_{0}(\lambda)=$ $\int_{0}^{\infty} r \cdot \lambda(r) d r$ converges and $\operatorname{Ric}_{q} \geq-(n-1) \cdot \lambda \circ r_{0}(q)$ at all points $q \in M^{n}$,

(i) the sectional curvatures are uniformly bounded from below by some (negative) constant, and

(ii) $M^{n}$ has diameter growth of order of $\left.r^{1 / n}\right)$ with respect to $p_{0}$.

Then all critical points of the distance function $r_{0}$ lie inside some large ball $B\left(p_{0}, R\right)$, which therefore is a deformation retract of $M^{n}$, and $M^{n}$ is homotopy equivalent to the interior of a compact manifold with boundary.

Let us illustrate our results in one example. Let $M\left(d_{1}, d_{2}\right)$ be the connected sum of infinitely many copies of $S^{d_{1}} \times S^{d_{2}}$, where $1 \leq d_{1} \leq d_{2}$ (see Figure 1). If $d_{1}=1$, the fundamental group grows exponentially and there cannot be a complete metric with Ric $\geq 0$ (cf. Proposition 1.3). Nothing can be said-using such a classical argument-for metrics with asymptotically nonnegative Ricci curvature. If $d_{1} \geq 2$ it has not been known so far whether or not $M\left(d_{1}, d_{2}\right)$ can carry any metric with Ric $\geq 0$ at all. It is easy to put complete Riemannian metrics on the manifolds $M\left(d_{1}, d_{2}\right)$ such that their diameter growth is of order $\mathscr{O}(1)$ ("bounded diameter"). By Theorem B these metrics cannot even have asymptotically nonnegative Ricci curvature, unless possibly their sectional curvature $K$ is not bounded away from $-\infty$.

Let us now discuss the additional hypothesis in Theorems A and B. Bounding the diameter growth seems to be a very natural condition. In fact, it is this 


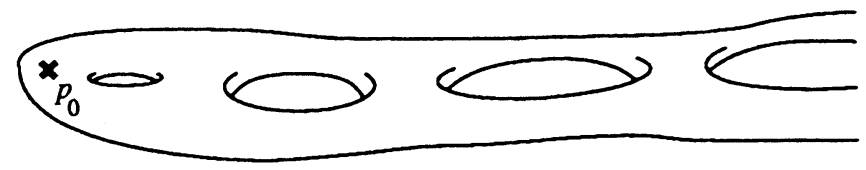

FIGURE 1

condition which is violated in the Sha-Yang examples. On these manifolds of infinite type the metric can be chosen to have diameter growth of order at most $\mathscr{O}\left(r^{2 / 3}\right)$. The condition also does not hold for the Bérard Bergery examples (finite homotopy type, diameter growth $\geq \mathscr{O}\left(r^{2 / 3}\right)$ ). However, it does hold in the large class of the Gromoll-Meyer examples. They all have even bounded diameter.

All these examples have sectional curvature bounded away from $-\infty$. Indeed this hypothesis appears to be a fairly weak assumption; it enters our arguments only in an integrated form (cf. Proposition 4.2).

In both theorems we have only claimed finite homotopy type for every single $M^{n}$, but not a uniform bound for a whole class of manifolds. Such a bound does not even exist for the numbers of homotopy types of compact manifolds with positive sectional curvature, as the examples of Wallach show [AW].

Nevertheless-as a consequence of Gromov's Betti numbers theorem (cf. [A, G]) - a uniform bound does exist for the homology types with coefficients in any field. This holds even for noncompact spaces with asymptotically nonnegative sectional curvature. However, such an estimate cannot hold for the class of compact manifolds with strictly positive Ricci curvature, according to examples in [ShY]. We do not know whether or not in our context a fixed lower sectional curvature bound and a specific diameter growth rate give rise to an a priori estimate for all the Betti numbers.

Many results on manifolds with $\mathrm{Ric} \geq 0$ are proven by volume comparison (cf. §1). These arguments are not sufficient to prove Theorems A and B. We need much stronger bounds for the distance function. In fact, the main result in $\S 2$ is a lower bound on the height of thin triangles involving just the lengths of their edges and a lower bound for the Ricci curvature (cf. Proposition 2.3 and Corollary 2.4). Here Toponogov's triangle comparison theorem is not required.

Our argument is modelled on the basic step in the proof of the Splitting Theorem; we calculate a bound on the Laplacian of certain distance functions and apply the maximum principle. In the case of the Splitting Theorem this bound is always zero; in our nonrigid situation the bound can-and will-take different values. This problem is dealt with in Theorem 2.1, which seems to be a new estimate on "subharmonic" Lipschitz functions.

In $\S 3$ we compute (as far as needed) the explicit bounds for the thin triangles. In particular, we analyze the asymptotic curvature condition so that in $\S 4$ we will be prepared to prove a new critical point lemma and deduce Theorems A and $B$. 


\section{Diameter growth AND Volume GROWTH}

Our first goal is to show that both notions, volume growth and diameter growth, can be used equally well to distinguish qualitatively between hyperbolic spaces and manifolds with nonnegative Ricci curvature. It is a direct consequence of the definitions that hyperbolic space has exponential volume growth as well as exponential diameter growth. Notice that we are considering the quantities $\operatorname{diam}\left(S\left(p_{0}, r\right), M^{n} \backslash B\left(p_{0}, \zeta \cdot r\right)\right)$, i.e., we have defined the relevant distance between two points $q_{1}, q_{2} \in S\left(p_{0}, r\right)$ as the infimum over the lengths of only those curves from $q_{1}$ to $q_{2}$ which lie inside $M^{n} \backslash B\left(p_{0}, \zeta \cdot r\right)$.

Proposition 1.1 (Linear diameter growth). Any complete Riemannian manifold with Ric $\geq 0$ has diameter growth of order $\mathscr{O}(r)$ with respect to any point $p_{0} \in M^{n}$.

This proposition is a direct consequence of Lemma 1.4 below. In order to make our point clear, let us state the corresponding result for volume growth next.

Proposition 1.2 (Polynomial volume growth). Let $M^{n}$ be a complete noncompact Riemannian manifold with $\mathrm{Ric} \geq 0$, and let $p_{0} \in M^{n}$ be arbitrary. Then,

(i) $\operatorname{vol} B\left(p_{0}, r\right) \leq \omega_{n} \cdot r^{n}$ for $r>0$, and

(ii) $\operatorname{vol} B\left(p_{0}, R\right) \geq \frac{1}{2}(R / r-1) \cdot \operatorname{vol} B\left(p_{0}, r\right)$ for $0<r<R$.

Here $\omega_{n}$ stands for the volume of the euclidean unit ball $B^{n}(1)$. This proposition completes our elementary comparison of volume and diameter growth. The second inequality is due to E. Calabi and S. T. Yau [CGT, Y2]. Since both statements are actually fairly direct consequences of the well-known relative volume comparison theorem, it is in fact easy to extend them-of course only up to some positive factors-to manifolds with asymptotically nonnegative Ricci curvature as we have defined them. (Notice that our condition is stronger than the condition of almost nonnegative Ricci curvature at infinity, which has been introduced in $\S 4$ of [CGT].) A result which does not extend is the following

Proposition 1.3 (Polynomial growth of $\pi_{1}$ ). Let $M^{n}$ be a complete Riemannian manifold with $\mathrm{Ric} \geq 0$. Then

$$
\#\left\{\alpha \in \pi_{1}\left(M^{n}\right) \mid\|\alpha\|_{\text {geo }} \leq r\right\} \leq \text { const } \cdot r^{n} .
$$

In particular, the first Betti number $b_{1}\left(M^{n}, \mathbf{R}\right)$ is bounded from above by $n$.

Here $\|\alpha\|_{\text {geo }}$ stands for the geometric norm taken w.r.t. some base point $\tilde{p}_{0}$ in the universal covering $\widetilde{M}^{n}$, i.e., $\|\alpha\|_{\text {geo }}=d\left(\tilde{p}_{0}, \tilde{\alpha} \cdot \tilde{p}_{0}\right)$, where $\tilde{\alpha}$ is the decktransformation representing $\alpha \in \pi_{1}\left(M^{n}\right)$. The proposition is proved by looking at the Dirichlet cell $\tilde{D}$ around $\tilde{p}_{0}$ and the action of the decktransformation group. Given $\rho_{0}>0$, one compares the volume of $\tilde{D} \cap B\left(\tilde{p}_{0}, \rho_{0}\right)$ to the volume of large balls $B\left(\tilde{p}_{0}, r\right)$ (cf. [CG2, M, An] for further results). 
Remark. Working with manifolds of asymptotically nonnegative Ricci curvature, one can in general at best pass to some finite covering, and this already weakens most decay conditions in relation to the degree of the covering. This makes it clear where the proof of Proposition 1.3 breaks down, when turning to manifolds with asymptotically nonnegative Ricci curvature. Of course, it is also easy to give a direct counterexample.

Before we begin with the proof of Proposition 1.1, let us recall the basic tool:

Relative Volume Comparison Theorem (R. Bishop [BC] and M. Gromov [GLP, MS]). Let $M^{n}$ be a complete Riemannian manifold with Ric $\geq(n-1) \cdot \kappa$, and let $q \in M^{n}$ be arbitrary. Then

$$
\frac{\operatorname{vol} B(q, r)}{\operatorname{vol} B(q, R)} \geq \frac{\operatorname{vol} B_{\kappa}(r)}{\operatorname{vol} B_{\kappa}(R)}, \quad \text { provided } 0 \leq r \leq R .
$$

Here $B_{\kappa}(r)$ denotes a ball of radius $r$ in the simply connected model space of constant curvature $\kappa$.

Lemma 1.4. Let $M^{n}$ be a complete Riemannian manifold with Ric $\geq 0$, and let $p_{0} \in M^{n}$. Then for all $r>0$,

$$
\operatorname{diam}\left(p_{0}, r\right) \leq 4 \xi \cdot(1+2 / \xi)^{n} \cdot r \quad \text { where } \xi=\frac{1}{2}(1-\zeta) .
$$

Proof. Pick a maximal family of points $q_{j} \in S\left(p_{0}, r\right)$ such that the balls $B_{j}=$ $B\left(q_{j}, \xi \cdot r\right)$ are disjoint. As $B_{j} \subset B\left(p_{0},(1+\xi) \cdot r\right) \subset B\left(q_{j},(2+\xi) \cdot r\right)$, it is standard to conclude-using the hypothesis Ric $\geq 0$ via the relative volume comparison theorem-that for all $j$,

$$
\left(\frac{\xi}{2+\xi}\right)^{n} \operatorname{vol} B\left(p_{0},(1+\xi) r\right) \leq \operatorname{vol} B_{j} \leq \operatorname{vol} B\left(p_{0},(1+\xi) r\right),
$$

and hence

$$
\#\left\{q_{j}\right\} \leq(1+2 / \xi)^{n} .
$$

The balls $B\left(q_{j}, 2 \xi r\right)$ cover $S\left(p_{0}, r\right)$, but they still do not intersect $B\left(p_{0}, \zeta r\right)$. In particular, if $B\left(q_{j}, 2 \xi r\right) \cap B\left(q_{j^{\prime}}, 2 \xi r\right) \neq \varnothing$, then the minimizing geodesic joining $q_{j}$ and $q_{j^{\prime}}$ has length less than $4 \xi r$, and hence does not intersect $B\left(p_{0}, \zeta r\right)$ either. Therefore the lemma follows directly by counting the number of balls $B\left(q_{j}, 2 \xi r\right)$, as in inequality (1.3).

The proofs of Proposition 1.3 and Lemma 1.4 illustrate how one can get some length control from volume estimates. This works since the standard volume estimates are for metric balls and involve the radius which is already a onedimensional quantity. We have actually proved more: if $M^{n}$ is a complete Riemannian manifold with Ric $\geq 0$, then for all $p_{0} \in M^{n}, r>0$, and all $\xi \in\left(0, \frac{1}{2}\right)$ the following inequality holds:

$$
\inf _{\Sigma} \sum_{j} \operatorname{diam}\left(\Sigma_{j}, M^{n} \backslash B\left(p_{0},(1-2 \xi) r\right)\right) \leq 4 \xi\left(1+\frac{2}{\xi}\right)^{n} \cdot r .
$$


Here the infimum is taken over all countable coverings $\Sigma=\left(\Sigma_{j}\right)$ of the distance sphere $S\left(p_{0}, r\right)$. It is necessary to allow that a single $\Sigma_{j}$ may consist of several connected components of $S\left(p_{0}, r\right)$. In this paper we are not going to compare diameter growth w.r.t. different base points in detail. One should certainly not expect a better statement than for volume growth; this notion is known to be independent of the base point only if the volume does not grow superexponentially. Without referring to Theorem B we do not know how to prove, in the case of asymptotically nonnegative Ricci curvature, that the diameter growth does not depend on the base point.

\section{THIN TRIANGLES}

In this section we present an inequality for thin triangles which requires only a lower bound for the Ricci curvature and allows us to generalize the basic argument in the proof of the Cheeger-Gromoll Theorem [CG2].

We begin with a fundamental estimate on "subharmonic" Lipschitz functions $f: M^{n} \rightarrow \mathbf{R}$. Bounds for the Laplacian of such a function will be formulated in terms of upper and lower barriers, just as in the proof of the splitting theorem given by J. Eschenburg and E. Heintze [EH]. An upper (resp. lower) barrier for $f$ at a point $q$ in the interior of the domain of $f$ is by definition a $C^{2}$-function $f_{q}$ defined on a given neighborhood $U_{q}$ of $q$ such that $f_{q} \geq f$ (resp. $f_{q} \leq f$ ) on $U_{q}$ and $f_{q}(q)=f(q)$.

This analytic result already requires the lower bound for the Ricci curvature. We use comparison with the standard model spaces $M_{\kappa}^{n}$ of constant curvature; in polar coordinates these spaces are usually described in terms of the functions:

and

$$
s_{\kappa}(t)= \begin{cases}\frac{1}{\sqrt{\kappa}} \sin \sqrt{\kappa} t, & \kappa>0, \\ t, & \kappa=0, \\ \frac{1}{\sqrt{-\kappa}} \sinh \sqrt{-\kappa} t, & \kappa<0,\end{cases}
$$

$$
c_{\kappa}(t)= \begin{cases}\cos \sqrt{\kappa} t, & \kappa>0, \\ 1, & \kappa=0, \\ \cosh \sqrt{-\kappa} t, & \kappa<0 .\end{cases}
$$

Our estimates in particular will involve the expression

$$
\varphi_{n, \kappa}(\rho, l)=\iint_{\rho \leq l \leq \tau \leq l}\left(\frac{s_{\kappa}(\tau)}{s_{\kappa}(t)}\right)^{n-1} d \tau d t,
$$

which is defined for $0<\rho \leq l$, provided $\kappa l \leq \pi^{2}$. Note that the radially symmetric function $\bar{h}(\bar{q}):=\varphi_{n, \kappa}(d(\bar{p}, \bar{q}), l)$ on the punctured ball $B(\bar{p}, l) \backslash\{\bar{p}\}$ in the model space $M_{\kappa}^{n}$ satisfies:

(i) $\Delta \bar{h}=1$ on $B(\bar{p}, l) \backslash\{\bar{p}\}$,

(ii) $\bar{h}(\bar{q})=0,\left.\operatorname{grad} \bar{h}\right|_{\bar{q}}=0$ for $\bar{q} \in \partial B(\bar{p}, l)$.

These two properties determine the function $\varphi_{n, \kappa}$. 
Theorem 2.1. Let $M^{n}$ be a complete Riemannian manifold, and let $f: B(p, R)$ $\subset M^{n} \rightarrow[0, \infty)$ be a Lipschitz function. Suppose that

(i) Ric $\geq(n-1) \cdot \kappa$ on $B(p, R)$,

(ii) $\operatorname{dil} f \leq C_{1}$,

(iii) $\Delta f \leq C_{2}$ in the sense that for all $q \in B(p, R)$ and all $\varepsilon>0$ there exists an upper barrier $f_{q, \varepsilon}$ for $f$ such that $\Delta f_{q, \varepsilon}(q) \leq C_{2}+\varepsilon$,

(iv) $f$ has a zero $z$ at distance $l:=d(p, z)<R$.

Then

$$
f(p) \leq \inf _{\rho \in(0, l)} C_{1} \rho+C_{2} \varphi_{n, \kappa}(\rho, l)=\Phi_{n}\left(\kappa, C_{1}, C_{2}, l\right)
$$

Remarks. (i) $C_{1} \geq 0$. Considering the zero $z$ of $f$, it is clear that $C_{2} \geq 0$ as well.

(ii) $\Phi_{n}\left(\kappa, C_{1}, 0, l\right)=\Phi_{n}\left(\kappa, 0, C_{2}, l\right)=0$.

(iii) Myers' theorem states that $\kappa \cdot l^{2} \leq \pi^{2}$. This inequality is precisely the condition under which $\Phi_{n}\left(\kappa, C_{1}, C_{2}, l\right)$ is well defined and depends continuously on its parameters.

Proof. Suppose the theorem is false. Using the continuity of $\Phi_{n}$ we can pick $\tilde{\kappa}<\kappa$ such that

$$
f(p)>\Phi_{n}\left(\tilde{\kappa}, C_{1}, C_{2}, l\right) \geq 0 \text { and } \tilde{\kappa} l^{2}<\pi^{2} .
$$

Similarly these inequalities persist when $C_{1}, C_{2}$, and $l$ are replaced by $\widetilde{C}_{1}=$ $C_{1}+\varepsilon, \widetilde{C}_{2}=C_{2}+\varepsilon$, and $\tilde{l}=l+\varepsilon$, provided $\varepsilon \in(0, R-l)$ is sufficiently small. We shall give a lower bound $h: B(p, R) \rightarrow[0, \infty)$ for $f$ such that $h$ is strictly positive on $B(p, \tilde{l})$. In particular, this yields $f(z) \geq h(z)>0$, contradicting hypothesis (iv).

In order to define $h$ let us consider the piecewise $C^{2}$-functions $\tilde{\varphi}_{\rho}:[0, R) \rightarrow$ $[0, \infty)$ defined by

$$
\tilde{\varphi}_{\rho}(d)= \begin{cases}\tilde{C}_{1} \cdot(\rho-d)+\tilde{C}_{2} \cdot \varphi_{n, \tilde{\kappa}}(d, \tilde{l}), & 0 \leq d \leq \rho, \\ \tilde{C}_{2} \cdot \varphi_{n, \tilde{\kappa}}(d, \tilde{l}), & \rho \leq d \leq \tilde{l}, \\ 0, & \tilde{l} \leq d<R .\end{cases}
$$

Since the map $d \mapsto \widetilde{C}_{2} \cdot \varphi_{n, \tilde{\kappa}}(d, \tilde{l})$ is strictly convex, there is precisely one $\rho_{0} \in(0, \tilde{l}]$ such that the function $\tilde{\varphi}_{\rho_{0}}$ is of class $C^{1}$. Clearly $\tilde{\varphi}_{\rho_{0}}(0)=$ $\Phi_{n}\left(\tilde{\kappa}, \widetilde{C}_{1}, \widetilde{C}_{2}, \tilde{l}\right)<f(p)$. We set

$$
h(q)=\tilde{\varphi}_{\rho_{0}}(d(p, q)) \text { for } q \in B(p, R) .
$$

It is clear that $\widetilde{C}_{1}, \widetilde{C}_{2}>0$, so $h$ is strictly positive in $B(p, \tilde{l})$ and vanishes outside this ball. It remains to show that

$$
f(q) \geq h(q) \text { for all } q \in B(p, \tilde{l}) .
$$

Since $f(p)>h(p)$, it follows directly from hypothesis (ii) that inequality (2.7) holds on $\overline{B\left(p, \rho_{0}\right)}$. In the annulus $A=B(p, \tilde{l}) \backslash \overline{B\left(p, \rho_{0}\right)}$ one can apply the 
maximum principle: If $f-h$ had a local minimum at some $q \in A$, then its upper barrier $f_{q, \varepsilon / 2}-h_{q}$ would have a local minimum at $q$ as well. Here $h_{q}$ denotes the lower barrier for $h$ constructed in Lemma 2.2 below. Therefore, $\Delta\left(f_{q, \varepsilon / 2}-h_{q}\right)(q) \leq C_{2}+\varepsilon / 2-\widetilde{C}_{2}<0$, a contradiction which shows that a local minimum of $f-h$ cannot exist in $A$.

Lemma 2.2. At any $q \in A$ the function $h$ defined in formula (2.6) has a lower barrier $h_{q}$ such that $\Delta h_{q}(q) \geq \widetilde{C}_{2}$.

Proof. Given $q \in A$, we pick a minimizing geodesic $\gamma$ from $p$ to $q$. Let $d=d(p, q)$ denote its length. For $\delta \in(0, d)$ we set

$$
h_{\delta}^{\gamma}(x)=\tilde{C}_{2} \cdot \varphi_{n, \kappa}(\delta+d(x, \gamma(\delta)), \tilde{l}) \text { for } x \in B(\gamma(\delta), \tilde{l}-\delta) .
$$

The map $\rho \mapsto C_{2} \cdot \varphi_{n, \tilde{\kappa}}(\rho, \tilde{l}), 0<\rho<\tilde{l}$, is decreasing. The triangle inequality implies that

$$
\begin{aligned}
& h_{\delta}^{\gamma}(q)=h(q), \\
& h_{\delta}^{\gamma}(x) \leq h(x) \text { for } x \in B(\gamma(\delta), \tilde{l}-\delta) .
\end{aligned}
$$

Since $\gamma$ is minimizing, its restriction to $[\delta, d]$ remains minimizing, even when it is extended a little beyond the endpoint $q=\gamma(d)$. Therefore the distance function $d_{\gamma(\delta)}(x)=d(\gamma(\delta), x)$ is differentiable in a neighborhood $U_{\delta}^{\gamma}$ of $\gamma(\delta, d]$, and so is the function $h_{\delta}^{\gamma}$. It is a standard fact that

$$
\begin{aligned}
& \left\|\operatorname{grad} d_{\gamma(\delta)}\right\|=1, \\
& \Delta d_{\gamma(\delta)} \leq(n-1) \frac{c_{\kappa}}{s_{\kappa}} \circ d_{\gamma(\delta)} \text { on } U_{\delta}^{\gamma} .
\end{aligned}
$$

We compute

$$
\begin{aligned}
\Delta h_{\delta}^{\gamma}(q)= & \left.\widetilde{C}_{2} \cdot \frac{\partial^{2}}{\partial \rho^{2}} \varphi_{n, \tilde{\kappa}}(\rho, \tilde{l})\right|_{\rho=d} \cdot\left\|\left.\operatorname{grad} d_{\gamma(\delta)}\right|_{q}\right\|^{2} \\
& +\left.\widetilde{C}_{2} \cdot \frac{\partial}{\partial \rho} \varphi_{n, \tilde{\kappa}}(\rho, \tilde{l})\right|_{\rho=d} \cdot \Delta d_{\gamma(\delta)}(q) \\
\geq & \widetilde{C}_{2}+(n-1) \tilde{C}_{2} \cdot\left(\frac{c_{\tilde{\kappa}}(d)}{s_{\tilde{\kappa}}(d)}-\frac{c_{\kappa}(d-\delta)}{s_{\kappa}(d-\delta)}\right) \cdot \int_{d}^{l}\left(\frac{s_{\tilde{\kappa}}(\tau)}{s_{\tilde{\kappa}}(d)}\right)^{n-1} d \tau .
\end{aligned}
$$

Since $\tilde{\kappa}<\kappa$, we can pick $\delta \in(0, d)$ so small that the expression on the righthand side is $\geq \widetilde{C}_{2}$. Because of formula (2.9) the function $h_{q}=h_{\delta}^{\gamma} \mid U_{\delta}^{\gamma}$ is the desired lower barrier at $q$ with $\Delta h_{q}(q) \geq \widetilde{C}_{2}$.

Theorem 2.1 has a direct geometric application. Let $\gamma$ be a minimizing geodesic joining two points $p_{0}, p_{1} \in M^{n}$. Given a third point $p \in M^{n}$ we set (cf. Figure 2)

$$
\begin{aligned}
& r_{i}(p)=d\left(p, p_{i}\right) \quad(i=0,1) \\
& l(p)=d(p, \gamma) \\
& e(p)=r_{0}(p)+r_{1}(p)-d\left(p_{0}, p_{1}\right) \quad \text { (the "excess function"). }
\end{aligned}
$$




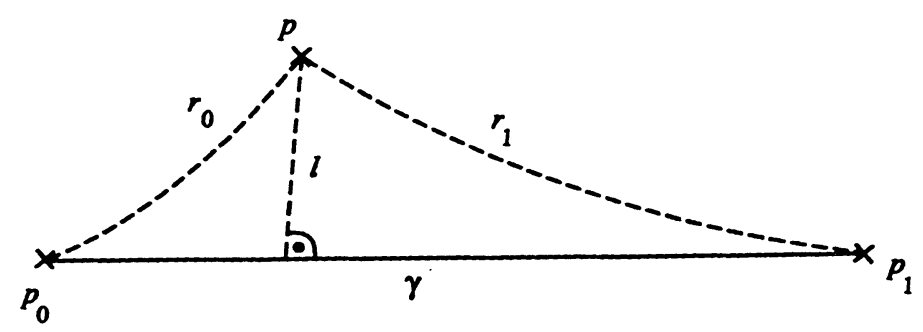

FIGURE 2

By the triangle inequality,

$$
0 \leq e(p) \leq 2 \cdot l(p)
$$

We are going to improve this inequality in the region where $l(p)$ is small, i.e., at points $p$ which are close to $\gamma$.

Proposition 2.3. Let $p, p_{0}, p_{1}$, and $\gamma$ be as above, and let $R>l(p)$. Suppose that $\mathrm{Ric} \geq(n-1) \kappa$ on $B(p, R)$. Moreover, we assume that the Laplacian of the excess function $e$ is bounded by some constant $C_{2}(R)$, in the sense that for all $q \in B(p, R)$ and all $\varepsilon>0$ there is an upper barrier $e_{q, \varepsilon}$ with $\Delta e_{q, \varepsilon}(q) \leq$ $C_{2}(R)+\varepsilon$. Then

$$
e(p) \leq \inf _{0<\rho<l(p)}\left\{2 \rho+C_{2}(r) \cdot \iint_{\rho \leq t \leq \tau \leq l(p)}\left(\frac{s_{\kappa}(\tau)}{s_{\kappa}(t)}\right)^{n-1} d \tau d t\right\}<2 l(p) .
$$

In particular, when $\kappa \leq 0$,

$$
e(p) \leq\left\{\begin{array}{l}
2 \cdot \frac{n-1}{n-2} \cdot\left(\frac{1}{2} C_{3} l^{n}\right)^{1 / n-1}, \quad n \geq 3 \\
C_{3} l^{2} \cdot\left(\frac{1}{1+\sqrt{1+C_{3}^{2} l^{2}}}+\ln \frac{1+\sqrt{1+C_{3}^{2} l^{2}}}{C_{3} l}\right), \quad n=2 .
\end{array}\right.
$$

Here we have set $l=l(p)$ and $C_{3}=\frac{1}{n} s_{\kappa l^{2}}(1) \cdot C_{2}(R)$.

Corollary 2.4. Let $p, p_{0}, p_{1}$, and $\gamma$ be as above. Assume that $M^{n}$ is a complete Riemannian manifold with Ric $\geq 0$. If $l(p)<\min \left\{r_{0}(p), r_{1}(p)\right\}$, then inequality (2.15) holds with

$$
C_{3}=\frac{n-1}{n} \cdot\left(\frac{1}{r_{0}(p)-l(p)}+\frac{1}{r_{1}(p)-l(p)}\right) .
$$

On the right-hand side of $(2.15)$ we see the factor $l^{n / n-1}$. The exponent $n / n-1$ occurs in the border line Sobolev embedding $L_{1}^{1}\left(\mathbf{R}^{n}\right) \rightarrow L^{n / n-1}\left(\mathbf{R}^{n}\right)$ for the very same reason: it makes both inequalities scale invariant.

When $n=2$, the exponent $n / n-1$ takes the value 2 . However, there is a logarithmic factor which makes our estimate (near $l=0$ ) even qualitatively 
weaker than the bound obtained from Toponogov's Theorem. But when $n \geq 3$ and we assume only that Ric $\geq 0$, Toponogov's Theorem does not apply.

Proof of Proposition 2.3. Since dile $\leq 2$, and since the excess function $e$ vanishes at the foot point $z$ of $p$ on $\gamma$, i.e., at a point in $B(p, R)$, inequality (2.14) is a direct consequence of Theorem 2.1. The proof of (2.15) is just computational. Using $t \cdot s_{\kappa t^{2}}(1)=s_{\kappa}(t)$ and $1 \leq s_{\kappa t^{2}}(1) \leq s_{\kappa \tau^{2}}(1) \leq s_{\kappa l^{2}}(1)$, we calculate that

$$
\begin{aligned}
2 \rho+ & C_{2}(R) \cdot \iint_{\rho \leq t \leq \tau \leq l(p)}\left(\frac{s_{\kappa}(\tau)}{s_{\kappa}(t)}\right)^{n-1} d \tau d t \\
& \leq 2 \rho+s_{\kappa l^{2}}(1)^{n-1} \cdot C_{2}(r) \cdot \int_{\rho}^{l} \int_{t}^{l}\left(\frac{\tau}{t}\right)^{n-1} d \tau d t \\
& \leq 2 \rho+\frac{1}{2} C_{3} \cdot\left(\rho^{2}-l^{2}+2 l^{n} \cdot \int_{\rho}^{l} t^{1-n} d t\right) .
\end{aligned}
$$

We regard the above right-hand side as a function $\Psi(\rho)$. It follows from inequality (2.14) that $e(p) \leq \inf \{\Psi(\rho) \mid 0<\rho<l\}$. The function $\Psi$ is convex, and the infimum is assumed at the unique $\rho_{0} \in(0, l)$ with $\Psi^{\prime}\left(\rho_{0}\right)=0$, or more explicitly,

$$
\rho_{0}^{n-1}=\frac{1}{2} C_{3} \cdot\left(l^{n}-\rho_{0}^{n}\right) \leq \frac{1}{2} C_{3} l^{n} .
$$

When $n \geq 3$, we conclude that

$$
\begin{aligned}
e(p) & \leq \Psi\left(\rho_{0}\right)=2 \rho_{0}+\frac{1}{2} C_{3} \cdot\left(\rho_{0}^{2}-\frac{n}{n-2} l^{2}+\frac{2}{n-2} l^{n} \rho_{0}^{2-n}\right) \\
& =2 \frac{n-1}{n-2} \rho_{0}+\frac{1}{2} \frac{2}{n-2} C_{3} \cdot\left(\rho_{0}^{2}-l^{2}\right) \\
& \leq 2 \frac{n-1}{n-2}\left(\frac{1}{2} C_{3} l^{n}\right)^{1 / n-1} .
\end{aligned}
$$

When $n=2$, we find

$$
e(p) \leq \Psi\left(\rho_{0}\right)=\rho_{0}+C_{3} l^{2} \cdot \ln \frac{l}{\rho_{0}} .
$$

In this case (2.15) follows by eliminating $\rho_{0}$ from the right-hand side using the quadratic equation in (2.18).

Proof of Corollary 2.4. Given $l(p)<R<\min \left\{r_{0}(p), r_{1}(p)\right\}$, we merely need to show that in $B(p, R)$ the Laplacian of the excess function $e$ is bounded by $C_{2}(R)=(n-1) /\left(r_{0}(p)-R\right)+(n-1) /\left(r_{1}(p)-R\right)$ in the sense of Proposition 2.3. In (2.15) we can then pass to the limit $R \rightarrow l(p)$. So let us pick minimizing geodesics $\gamma_{0}, \gamma_{1}$ from $p_{0}, p_{1}$ to some point $q \in B(p, R)$. We set

$$
e_{q}^{\delta}(x)=2 \delta+d\left(\gamma_{0}(\delta), x\right)+d\left(\gamma_{1}(\delta), x\right)-d\left(p_{0}, p_{1}\right) \quad \text { for } x \in M^{n},
$$


where $\delta$ varies between 0 and $\min \left\{r_{0}(p), r_{1}(p)\right\}-R$. Each function $e_{q}^{\delta}$ is differentiable when restricted to a suitable neighborhood $U_{q}^{\delta}$ of $q$. Indeed, $e_{q}^{\delta} \mid U_{q}^{\delta}$ is an upper barrier for $e$ at $q$ such that

$$
\Delta e_{q}^{\delta}(x) \leq \frac{n-1}{r_{0}(q)-\delta}+\frac{n-1}{r_{1}(q)-\delta}<\frac{n-1}{r_{0}(p)-R-\delta}+\frac{n-1}{r_{1}(p)-R-\delta}
$$

(cf. formula (2.10)). Thus given $\varepsilon>0$, we can choose $\delta(\varepsilon)>0$ so small that $\Delta e_{q}^{\delta(\varepsilon)}(q) \leq C_{2}(R)$, as required.

\section{EXPLICIT ESTIMATES FOR MANIFOLDS WITH ASYMPTOTICALLY NONNEGATIVE RICCI CURVATURE}

In this section we are going to determine explicit bounds for thin triangles in our more general situation.

Proposition 3.1. Let $M^{n}$ be a complete Riemannian manifold of dimension $n \geq$ 3 , and let $p, p_{0}, p_{1}$, and $\gamma$ be as in (2.12) (cf. Figure 2). Suppose $L:=$ $d\left(p_{0}, p_{1}\right) \geq 2 \cdot r_{0}(p)$ and, moreover, that there exists a nonincreasing function $\lambda:[0, \infty) \rightarrow[0, \infty)$ such that $C_{0}(\lambda)=\int_{0}^{\infty} r \lambda(r) d r$ converges and $\mathrm{Ric}_{q} \geq$ $-(n-1) \cdot \lambda \circ r_{0}(q)$ at all points $q \in M^{n}$. Then the height of the triangles can be bounded from below in terms of $r_{0}(p)$ and the excess $e(p)$ :

$$
d(p, \gamma) \geq \min \left\{\frac{1}{6} r_{0}(p), \frac{r_{0}(p)}{\sqrt{1+8 C_{0}(\lambda)}}, C_{4} \cdot r_{0}(p)^{1 / n} \cdot(2 e(p))^{1-1 / n}\right\}
$$

where

$$
C_{4}=C_{4}(n, \lambda)=\frac{4}{17} \frac{n-2}{n-1}\left(\frac{5}{1+\sqrt{1+8 C_{0}(\lambda)}}\right)^{1 / n} .
$$

Remarks. (i) For manifolds with nonnegative Ricci curvature we have-as a direct consequence of Corollary 2.4-the stronger estimate

$$
d(p, \gamma) \geq \frac{1}{2} \min \left\{r_{0}(p), \frac{n-2}{n-1} \cdot r_{0}(p)^{1 / n} \cdot e(p)^{1-1 / n}\right\} .
$$

(ii) Since $C_{4}(2, \lambda)=0$, the proposition holds trivially for 2-manifolds so that we need not explicitly exclude this case in subsequent applications. It has already been explained after Corollary 2.4 that more reasonable estimates in the two-dimensional case should be based on Toponogov's Theorem; we are not going to state them here.

The convergence of the integral $C_{0}(\lambda)$, which is a hypothesis of the proposition, is essentially a decay condition on the lower curvature bound. Roughly speaking, this bound must tend to zero a little quicker than const $\cdot r_{0}(p)^{-2}$. 
More precisely:

Lemma 3.2. Let $\lambda:[0, \infty) \rightarrow[0, \infty)$ be a monotonic function such that $C_{0}(\lambda)=$ $\int_{0}^{\infty} r \lambda(r) d r$ converges. Then the monotonic functions

$$
\lambda_{1}(r)=\int_{r}^{\infty} \lambda(t) d t \text { and } \lambda_{2}(r)=\int_{r}^{\infty} \lambda_{1}(t) d t
$$

exist, and moreover,

$$
r^{2} \lambda \leq 2 C_{0}(\lambda) \text { and } r \lambda_{1}(r) \leq C_{0}(\lambda) \text { for all } r>0 \text {. }
$$

This lemma, which has been proved in Chapter II of [A], will be useful in deducing Proposition 3.1 from Proposition 2.3. However, before we can actually give this argument, we need to know more about the analysis of the decay condition. Let us consider the Riccati equation

$$
u^{\prime}(r)=u(r)^{2}-\lambda(r) \text {. }
$$

For any $L>0$ there are unique solutions $u_{L}^{0}:(0, L] \rightarrow[0, \infty)$ such that $u_{L}^{0}(L)=0$ and $u_{L}^{\infty}:(0, L) \rightarrow(0, \infty)$ such that $u_{L}(r) \rightarrow+\infty$ for $r \rightarrow L$.

Lemma 3.3. If $C_{0}(\lambda)$ converges, then:

(i) The solutions $u_{L}^{0}(r)$ of (3.4) depend monotonically on $r$ and on the parameter $L$. They are bounded by $\min \left\{\lambda_{1}(r), \sqrt{\lambda(r)}\right\}$, and hence in the limit $L \rightarrow \infty$, they converge to a nonincreasing solution $u_{\infty}:(0, \infty) \rightarrow[0, \infty)$.

(ii) The solutions $u_{L}^{\infty}(r)$ also converge monotonically in $L$ and uniformly on compact subsets of $(0, \infty)$ to the solution $u_{\infty}$. When $0<r<L$, the following inequalities hold:

$$
u_{\infty}(r)<u_{L}^{\infty}(r) \leq u_{\infty}(r)+\frac{1}{L-r} \leq \min \left\{\lambda_{1}(r), \sqrt{\lambda(r)}\right\}+\frac{1}{L-r} .
$$

Proof. Part (i) of this lemma has also been proved in Chapter II of [A], where the condition $C_{0}(\lambda)<\infty$ has been analyzed in detail. Anyway, the common upper bound for the functions $u_{L}^{0}$ as well as their monotonicity is obtained by a simple comparison of first order differential equations. In order to prove part (ii), let us substitute $u(r)=u_{\infty}(r)+v(r)^{-1}$ into equation (3.4). We see that the function $v$ satisfies

$$
v^{\prime}(r)=-1-2 u_{\infty}(r) \cdot v(r) .
$$

Since any positive initial value $v_{0}$ decays to zero within finite time, we conclude that any solution $u(r)$ which exceeds $u_{\infty}(r)$ at some point cannot exist globally on $(0, \infty)$ and is in fact some $u_{L}^{\infty}$. Equation (3.6) also implies that $u_{\infty}(r)<$ $u_{L}^{\infty}(r) \leq u_{\infty}(r)+1 /(L-r)$.

For any $\delta \geq 0$ let $w_{\delta}^{\infty}:(\delta, \infty) \rightarrow(0, \infty)$ be the unique nonincreasing solution of

$$
w^{\prime}(r)+w(r)^{2}-\lambda(r)=0,
$$

with initial data given by $\lim _{r \rightarrow \delta} w(r)=+\infty$. 
Lemma 3.4. Suppose that $C_{0}(\lambda)$ converges. Then for all $r>0$ and all $\varepsilon>0$ there is some $\delta(\varepsilon, r)>0$ such that

$$
w_{\delta}^{\infty}(r) \leq \frac{1}{2 r} \cdot\left(1+\sqrt{1+8 C_{0}(\lambda)}\right)+\varepsilon \text { for } 0 \leq \delta \leq \delta(\varepsilon, r) .
$$

Proof. Set $a=\frac{1}{2}\left(1+\sqrt{1+8 C_{0}(\lambda)}\right)$. By Lemma 3.2 it is clear that $\lambda(r) \leq$ $-a(1-a) r^{-2}$ for all $r>0$, and so $v^{\prime}(r)+v(r)^{2}+a(1-a) r^{-2}=0$ is a comparison equation for (3.7). Its generic solution is

$$
v_{\delta}^{\infty}(r)=\frac{a}{r}+\frac{2 a-1}{r} \frac{\delta^{2 a-1}}{r^{2 a-1}-\delta^{2 a-1}} .
$$

Standard comparison arguments yield $w_{\delta}^{\infty}(r) \leq v_{\delta}^{\infty}(r)$ for $r>\delta \geq 0$.

In subsequent arguments the Riccati equations (3.4) and (3.7), which have been analyzed above, will be used as (one-dimensional) comparison equations in the following geometric context: let $d_{\tilde{p}}$ denote the distance function to some point $\tilde{p} \in M^{n}$, and let $c$ be a unit speed geodesic of finite length which begins at $c(0)=\tilde{p}$ and which does not intersect the cut locus $C_{\tilde{p}}$ of $\tilde{p}$. Then $d_{\tilde{p}}$ is differentiable along $c$ except at $\tilde{p}$ itself, and its Hessian, viewed as a symmetric, $(1,1)$-tensor, satisfies

$$
\nabla_{c^{\prime}} \operatorname{Hess} d_{\tilde{p}}+\left(\operatorname{Hess} d_{\tilde{p}}\right)^{2}+R\left(\cdot, c^{\prime}\right) c^{\prime}=0
$$

and hence the differential inequality

$$
d_{c^{\prime}}\left(\frac{1}{n-1} \Delta d_{\hat{p}}\right)+\left(\frac{1}{n-1} \Delta d_{\tilde{p}}\right)^{2}+\frac{1}{n-1}\left\langle\operatorname{Ric} c^{\prime}, c^{\prime}\right\rangle \leq 0 .
$$

Proof of Proposition 3.1. It is sufficient to consider the case where

$$
l(p)<\tilde{r}_{0}(p)=\min \left\{\frac{1}{6} r_{0}(p), \frac{r_{0}(p)}{\sqrt{1+8 C_{0}(\lambda)}}\right\} .
$$

Let us choose $l(p)<R<\tilde{r}_{0}(p)$. Our goal is to apply Proposition 2.3 to the triangle $p_{0}, p_{1}, p$. The lower bound $\kappa$ on the Ricci curvature in the ball $B(p, R)$ can be controlled by means of Lemma 3.3; it follows that

$$
\begin{aligned}
s_{\kappa l(p)^{2}}(1) & \leq \frac{5}{6} \frac{\sqrt{1+8 C_{0}(\lambda)}}{\sqrt{2 C_{0}(\lambda)}} \sinh \frac{6}{5} \frac{\sqrt{2 C_{0}(\lambda)}}{\sqrt{1+8 C_{0}(\lambda)}} \\
& <\frac{5}{3} \sinh \frac{3}{5}<\frac{17}{16} .
\end{aligned}
$$

In order to be able to use Proposition 2.3, it is therefore sufficient to give a weak upper bound for the Laplacian of the excess function $e$ on the ball $B(p, R)$. Upper barriers at some point $q \in B(p, R)$ can be defined as in the proof of Corollary 2.4; we select minimizing unit speed geodesics $\gamma_{0}, \gamma_{1}$ from $p_{0}, p_{1}$ to $q$. For small $\delta>0$ and all $x \in B(p, R)$ we define

$$
e_{q}^{\delta}(x)=2 \delta+d\left(\gamma_{0}(\delta), x\right)+d\left(\gamma_{1}(\delta), x\right)-L
$$


Again, the point $q=\gamma_{0}\left(r_{0}(q)\right)=\gamma_{1}\left(r_{1}(q)\right)$ lies neither on the cut locus of $\gamma_{0}(\delta)$ nor on the cut locus of $\gamma_{1}(\delta)$. The distance functions $d_{\gamma_{0}(\delta)}$ and $d_{\gamma_{1}(\delta)}$ are differentiable along the curves $\delta_{0} \mid\left(\delta, r_{0}(q)\right]$ and $\gamma_{1} \mid\left(\delta, r_{1}(q)\right]$, respectively. In particular, the differential inequality $\left(3.10^{\prime}\right)$ holds along both these geodesics.

Since Ric| $\left.\right|_{\gamma_{0}(r)} \geq-(n-1) \lambda(r)$, comparison of $\left(3.10^{\prime}\right)$ with the Riccati equation (3.7) yields

$$
\Delta d_{\gamma_{0}(\delta)} \leq(n-1) w_{\delta}^{\infty}\left(r_{0}(q)\right) .
$$

Since $\lambda$ is supposed to be nonincreasing, it follows from the triangle inequality that $\left.\mathrm{Ric}\right|_{\gamma_{1}(L-r)} \geq-(n-1) \lambda(r)$. As the parametrization has been reversed, the differential inequality for $\Delta d_{\gamma_{1}(\delta)} /(n-1)$ must be compared to the Riccati equation (3.4) rather than (3.7). We conclude that

$$
\Delta d_{\gamma_{1}(\delta)}(q) \leq(n-1) \cdot u_{L-\delta}^{\infty}\left(L-r_{1}(q)\right)
$$

Our estimates above verify that each function $e_{q}^{\delta}$ is an upper barrier for the excess function $e$ at $q$ when restricted to a suitable neighborhood $U_{q}^{\delta}$ of this point. It satisfies

$$
\Delta e_{q}^{\delta}(q) \leq(n-1) \cdot\left[w_{\delta}^{\infty}\left(r_{0}(q)\right)+u_{L-\delta}^{\infty}\left(L-r_{1}(q)\right)\right] .
$$

In the limit $\delta \rightarrow 0$ the right-hand side of $(3.15)$ converges to

$$
(n-1)\left[w_{0}^{\infty}\left(r_{0}(q)\right)+u_{L}^{\infty}\left(L-r_{1}(q)\right)\right] .
$$

Therefore Proposition 2.3 yields

$$
e(p) \leq 2 \frac{n-1}{n-2} \cdot\left(\frac{1}{2} C_{3} l(p)^{n}\right)^{1 / n-1},
$$

where

$$
C_{3}=\frac{n-1}{n} \cdot\left(\frac{17}{16}\right)^{n-1} \cdot\left(w_{0}^{\infty}\left(r_{0}(p)-R\right)+\sup _{|r| \leq R} u_{L}^{\infty}\left(L-r_{1}(p)-r\right)\right) .
$$

Of course, this estimate can be slightly improved by taking the limit $R \rightarrow l(p)$. From 3.2 and 3.3 we conclude that

$$
\begin{aligned}
\sup _{|r| \leq l} u_{L}^{\infty}\left(L-r_{1}(p)-r\right) & \leq \sup _{|r| \leq l}\left\{u_{\infty}\left(L-r_{1}(p)-r\right)+\frac{1}{r_{1}(p)+r}\right\} \\
& \leq \sup _{|r| \leq l}\left\{\frac{\sqrt{2 C_{0}(\lambda)}}{L-r_{1}(p)-r}+\frac{1}{r_{1}(p)+r}\right\} \\
& \leq \sup _{|r| \leq l}\left\{\frac{3}{2} \cdot \frac{\sqrt{1+8 C_{0}(\lambda)}}{2 r_{0}(p)-3 r}+\frac{1}{r_{0}(p)+r}\right\} \\
& \leq\left(\frac{6}{7}+\sqrt{1+8 C_{0}(\lambda)}\right) \frac{1}{r_{0}(p)} .
\end{aligned}
$$


Here we have used the inequalities $L-r_{1}(p) \geq r_{0}(p)-2 l(p) \geq \frac{2}{3} r_{0}(p)$ and $r_{1}(p) \geq L-r_{0}(p) \geq r_{0}(p)$, and the assumption $l(p)<\frac{1}{6} r_{0}(p)$ itself. Similarly, Lemma 3.4 yields that

$$
w_{0}^{\infty}\left(r_{0}(p)-l(p)\right) \leq \frac{3}{5}\left(1+\sqrt{1+8 C_{0}(\lambda)}\right) \frac{1}{r_{0}(p)} .
$$

Combining (3.16) through (3.18), we obtain

$$
r_{0}(p) \cdot(2 e(p))^{n-1} \leq\left(\frac{17}{4} \frac{n-1}{n-2} l(p)\right)^{n} \cdot \frac{8}{5} \cdot \frac{2}{17} \frac{n-2}{n}\left(1+\sqrt{1+8 C_{0}(\lambda)}\right),
$$

and the proposition is proved.

\section{A NEW CRITICAL POINT LEMMA}

Before we can establish our main theorem, we need to recall another concept: Fix a point $p_{0} \in M^{n}$ and consider the distance function $r_{0}(p)=d\left(p_{0}, p\right)$ on $M$. A point $p \in M^{n}$ is a critical point of $r_{0}$ if and only if for any nonzero tangent vector $v \in T_{p} M^{n}$ there is a minimizing geodesic $\gamma_{0}$ to $p_{0}$ such that $\Varangle\left(\gamma_{0}^{\prime}(0), v\right) \leq \pi / 2$. It is easy to define a continuous gradient-like vector field $\tilde{v}$ on the complement of the set of critical points of $r_{0}$, which gives rise to the

Isotopy Lemma (cf. [GS, G]). Let $0<\rho_{1}<\rho_{2}$, and let $C$ be a connected component of $\overline{B\left(p_{0}, \rho_{2}\right)} \backslash B\left(p_{0}, \rho_{1}\right)$. Let $U$ be an open neighborhood of $C$. Suppose that $C$ contains no critical point of $r_{0}$. Then there exists

(i) an isotopy from $\overline{B\left(p_{0}, \rho_{2}\right)}$ to $\overline{B\left(p_{0}, \rho_{2}\right)} \backslash C$ which is the identity map outside of $U$, and

(ii) an isotopy from $M^{n} \backslash B\left(p_{0}, \rho_{2}\right)$ to $\left(B\left(p_{0}, \rho_{2}\right) \cup C\right)$ which is the identity map outside $U$.

The hypothesis on sectional curvature enters the proof of Theorem B through the following

Lemma 4.1 (Critical Point Lemma). Let $M^{n}$ be a complete Riemannian manifold with base point $p_{0}$, and let $p \in M^{n}$ be a critical point of $r_{0}$. Suppose:

(0) There is a nonincreasing function $\lambda:[0, \infty) \rightarrow[0, \infty)$ such that $C_{0}(\lambda)=$ $\int_{0}^{\infty} r \lambda(r) d r$ converges and that $\mathrm{Ric}_{q} \geq-(n-1) \cdot \lambda \circ r_{0}(q)$ at all points $q \in M^{n}$.

(i) The sectional curvatures of $M^{n}$ are bounded from below by $-\Lambda^{2}$, where $\Lambda$ is some positive constant, and

(ii) $r_{0}(p) \geq R_{0}=\max \left\{\frac{3}{2 \Lambda}, \frac{4}{17 \Lambda}\left(1+\sqrt{1+8 C_{0}(\lambda)}\right)\right\}$.

Then any minimizing geodesic from $p_{0}$ to a point in $B\left(p, C_{4} \Lambda^{-1+1 / n} r_{0}(p)^{1 / n}\right)$, when extended beyond its endpoint, will meet the cut locus $C_{p_{0}}$ of $p_{0}$, before its length exceeds $2 r_{0}(p)$.

Here

$$
C_{4}=C_{4}(n, \lambda)=\frac{4}{17} \frac{n-2}{n-1}\left(\frac{5}{\sqrt{1+8 C_{0}(\lambda)}}\right)^{1 / n},
$$

as in Proposition 3.1. 
Proof. Assume on the contrary that there is a minimizing geodesic $\gamma$ from $p_{0}$ to some point $p_{1} \in S\left(p_{0}, 2 r_{0}(p)\right)$ such that $d(p, \gamma) \leq C_{4} \Lambda^{-1+1 / n} r_{0}(p)^{1 / n}$. Because of hypothesis (ii) we know that

$$
\min \left\{\frac{1}{6} r_{0}(p), \frac{r_{0}(p)}{\sqrt{1+8 C_{0}(\lambda)}}\right\} \geq C_{4} \cdot \Lambda^{-1+1 / n} \cdot r_{0}(p)^{1 / n}
$$

Therefore Proposition 3.1 implies that $e(p) \leq 1 / 2 \Lambda$.

On the other hand, we can reason as in the proof of the standard critical point lemma: Let $\gamma_{1}$ be a minimizing unit speed geodesic from $p$ to $p_{1}$. Since $p$ is a critical point of $r_{0}$, there exists a minimizing geodesic $\gamma_{0}$ from $p$ to $p_{0}$ such that $\Varangle\left(\gamma_{0}^{\prime}(0), \gamma_{1}^{\prime}(0)\right) \leq \pi / 2$. Let us consider the points $\tilde{p}_{0}=\gamma_{0}(\rho)$ and $\tilde{p}_{1}=$ $\gamma_{1}(\rho)$, where $\rho=5 / 4 \Lambda$. The triangle inequality implies $e(p)=2 \rho-d\left(\tilde{p}_{0}, \tilde{p}_{1}\right)$. Applying Toponogov's Theorem and the Law of Cosines to the isosceles triangle $\tilde{p}_{0} p \tilde{p}_{1}$, we obtain that $\cosh \Lambda d\left(p_{0}, p_{1}\right) \leq \cosh ^{2} \Lambda \rho$. Altogether,

$$
e(p) \geq 2 \rho-\Lambda^{-1} \operatorname{arccosh}\left(\cosh ^{2} \Lambda \rho\right)>\frac{1}{2 \Lambda} .
$$

This contradicts the upper bound for the excess obtained from Proposition 3.1, and the lemma is proved.

Remarks. (i) Recall that the standard critical point lemma is proved by applying Toponogov's Theorem twice (cf. [G, GS]). We have replaced one of these steps by our estimate in Proposition 3.1. This way we can make use of a lower bound for Ricci curvature, which in our case is quantitatively considerably stronger than the lower bound for sectional curvature. The price paid for working with the weaker notion of curvature is that we can only control the height $d(p, \gamma)$ of the triangle $p_{0} p p_{1}$ from below, rather than its angle at $p_{0}$.

(ii) Since the function $2 \rho-\Lambda^{-1} \cdot \operatorname{arccosh}\left(\cosh ^{2} \Lambda \rho\right)$ is monotonically increasing in $\rho$ and bounded by $\Lambda^{-1} \ln (2)$, it is clear that we are not losing much when choosing $\rho$ to be $5 / 4 \Lambda$ in the proof of the lemma. We emphasize that Toponogov's Theorem is only needed to get a uniform estimate for the excess of the a priori bounded triangles $\tilde{p}_{0} p \tilde{p}_{1}$. This suggests that a lower bound for sectional curvature which we have required in Lemma 4.1 might just be a technical hypothesis. It is an open question whether there is a critical point lemma which involves only a lower bound on the Ricci curvature.

Roughly speaking, Lemma 4.1 confines the size of the set of critical points. This restriction, which is nontrivial on all complete Riemannian manifolds, can be made more explicit for spaces satisfying a suitable diameter growth condition. 
Proposition 4.2. Let $M^{n}$ be a complete Riemannian manifold with base point $p_{0}$. Suppose that:

(0) There is a nonincreasing function $\lambda:[0, \infty) \rightarrow[0, \infty)$ such that $C_{0}(\lambda)=$ $\int_{0}^{\infty} r \lambda(r) d r$ converges and $\mathrm{Ric}_{q} \geq-(n-1) \lambda \circ r_{0}(q)$ at all points $q \in$ $M^{n}$.

(i) The sectional curvatures are uniformly bounded from below by $-\Lambda^{2}$, where $\Lambda$ is some positive constant, and

(ii) there exists $R_{1}>0$ such that $\operatorname{diam}\left(p_{0}, r\right)<C_{4}(n, \lambda) \Lambda^{-1+1 / n} r^{1 / n}$ for all $r>R_{1}$.

Then all critical points of $r_{0}$ are contained in the union of $\overline{B\left(p_{0}, R_{2}\right)}$ and all bounded components $K$ of $M^{n} \backslash \overline{B\left(p_{0}, R_{2}\right)}$, where

$$
R_{2}=\max \left\{R_{1}, \frac{3}{2 \Lambda}, \frac{4}{17 \Lambda}\left(1+\sqrt{1+8 C_{0}(\lambda)}\right)\right\} .
$$

Notice that the constant $C_{4}(n, \lambda)$ contains a factor $(n-2) /(n-1)$, and so hypothesis (ii) implies that $M^{n}$ has dimension $n \geq 3$.

Proof. Assume on the contrary that there is a critical point $p$ of $r_{0}$ which lies in an unbounded component $C$ of $M^{n} \backslash \overline{B\left(p_{0}, R_{2}+\delta\right)}$ for some $\delta>0$. By the Hopf-Rinow Theorem there exists a sequence of points $p_{j} \in C, 1 \leq j<\infty$, such that $r_{0}\left(p_{j}\right) \rightarrow \infty$ in the limit $j \rightarrow \infty$. Let $\gamma_{j}$ be a minimizing geodesic from $p_{0}$ to $p_{j}$. It is a standard fact that a subsequence of these geodesics $\gamma_{j}$ converges towards a ray $\gamma$ emanating from $p_{0}$.

Clearly $\gamma\left(R_{2}+\delta, \infty\right) \subset C$. On the other hand, our critical point lemma implies that $d(p, \gamma) \geq C_{4}(n, \lambda) \cdot \Lambda^{-1+1 / n} r_{0}(p)^{1 / n}$. Using hypothesis (ii) we conclude that $p$ and $\gamma \circ r_{0}(p)$ lie in different connected components of the distance sphere $S\left(p_{0}, r_{0}(p)\right)$.

From the way the ray $\gamma$ has been constructed, it is clear that there is a continuous curve $c:[0,1] \rightarrow C$ such that $c(0)=\gamma \circ r_{0}(p)$ and $c(1)=p$. Let $\Sigma(t)$ be the connected component of $S\left(p_{0}, r_{0}(c(t))\right)$ which contains $\gamma \circ r_{0}(c(t))$. Consider the set

$$
A=\{t \in[0,1] \mid c(t) \in \Sigma(t)\} .
$$

Now $0 \in A, 1 \notin A$, and $A \subset[0,1]$ is a closed subset. Our indirect proof will be accomplished by deriving the contradiction that $A$ is an open subset of $[0,1]$ as well. For this purpose let us pick some $t \in A$. By hypothesis (ii), there is an $\varepsilon$-neighborhood $U_{\varepsilon} \Sigma(t)=\left\{x \in M^{n} \mid d(x, \Sigma(t)) \leq \varepsilon\right\}$ of $\Sigma(t)$ such that

$$
\operatorname{diam} U_{\varepsilon} \Sigma(t)<C_{4}(n, \lambda) \cdot \Lambda^{-1+1 / n} \cdot\left(r_{0} \circ c(t)-\varepsilon\right)^{1 / n} .
$$

Choosing $\rho>0$ sufficiently small, we may assume that the intersection of the annulus $A(t, \rho)=\overline{B\left(p_{0}, r_{0} \circ c(t)+\rho\right)} \backslash B\left(p_{0}, r_{0} \circ c(t)-\rho\right)$ and $U_{\varepsilon} \Sigma(t)$ is a connected component of $A(t, \rho)$. In light of Lemma 4.1, inequality (4.4) implies that the component $A(t, \rho) \cap U_{\varepsilon} \Sigma(t)$ contains no critical point of $r_{0}$, 


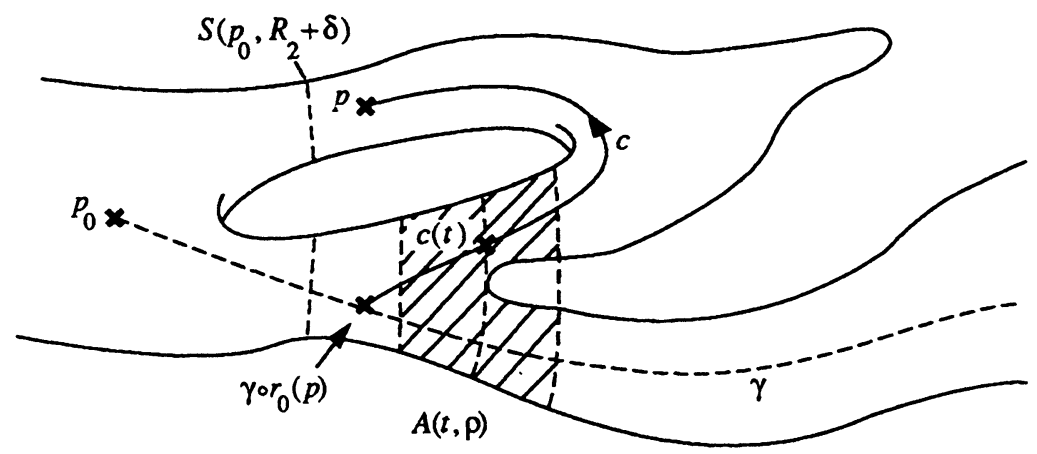

FIGURE 3. This figure depicts the basic problem which has been taken care of in the proof of Proposition 4.2. Our reasoning is essentially that, on the one hand, the annulus $A(t, \rho)$ must contain a critical point of $r_{0}$, since the curve $c(t)$ leaves the component $\Sigma(t)$ of the distance sphere $S\left(p_{0}, r_{0} \circ c(t)\right)$, while, on the other hand, it cannot contain such a point by the estimate given in Lemma 4.1.

and therefore the Isotopy Lemma applies to this piece of the annulus. In this context let us consider an open neighborhood $U(t)$ of $t$ in $[0,1]$ such that $\Sigma\left(t^{\prime}\right) \subset A(t, \rho) \cap U_{\varepsilon} \Sigma(t)$ for all $t^{\prime} \in U(t)$. The isotopies of the set $A(t, \rho) \cap$ $U_{\varepsilon} \Sigma(t)$ in its neighborhood $U_{\varepsilon} \Sigma(t)$, which we have obtained above, show that $c\left(t^{\prime}\right) \in \Sigma\left(t^{\prime}\right)$ for all $t^{\prime} \in U(t)$. Hence $U(t) \subset A$, i.e., $t$ is an interior point of the subset $A \subset[0,1]$.

Proof of Theorem B. In dimension $n=2$, we are just dealing with asymptotically nonnegative sectional curvature, and Theorem B turns out to be an easy corollary of the proof of the Betti number theorem as given in [A].

In the general case when $M^{n}$ has dimension $n \geq 3$ it is evident that there exists some radius $R_{1}>0$ such that $\operatorname{diam}\left(p_{0}, r\right)<C_{4}(n, \lambda) \Lambda^{-1+1 / n} r^{1 / n}$ for all $r>R_{1}$, simply because we are assuming that $M^{n}$ has diameter growth of order $\sigma\left(r^{1 / n}\right)$. Hence it follows from Proposition 4.2 that all critical points of $r_{0}$ are contained in some large ball $B\left(p_{0}, R\right)$. Notice that we do not claim that $M^{n} \backslash \overline{B\left(p_{0}, R_{2}\right)}$ has only finitely many bounded connected components $K$; this is only true for the complement of a generic closed ball. Anyway, all but finitely many of the connected components $K$ are contained in $B\left(p_{0}, 2 R_{2}\right)$, and this is all we have used.

Since $M^{n}$ is connected, the other assertions in Theorem B follow now by standard isotopy arguments.

Theorem A is a special case of Theorem B, and so we have proved it as well. Finally let us point out that, in case one only wants to deal with manifolds of nonnegative Ricci curvature, the isotopy arguments in the proof of Proposition 
4.2 are not needed. Instead we could refer to the following

Proposition 4.3. Let $M^{n}$ be a complete Riemannian manifold with $\mathrm{Ric} \geq 0$. Then

$$
\# \operatorname{im}\left(j_{*}^{\Omega}: \pi_{1}\left(M^{n}\right) \rightarrow \pi_{1}\left(M^{n} /\left(M^{n} \backslash \Omega\right)\right) \leq 2\right.
$$

for any bounded domain $\Omega \subset M^{n}$. Moreover, given any ball $B\left(p_{0}, r\right) \subset M^{n}$, the boundary of each component of the complement $M^{n} \backslash B\left(p_{0}, r\right)$ must be connected. Proof. As any complete manifold with two or more ends contains a line, we conclude from the Cheeger-Gromoll splitting theorem that the universal covering $\widetilde{M}^{n}$ has at most two ends. Now (4.5) follows by counting the preimages in $\widetilde{M}^{n}$ of the point $\left[M^{n} \backslash \Omega\right]$ in the quotient space $M^{n} /\left(M^{n} \backslash \Omega\right)$, using the following commutative diagram:

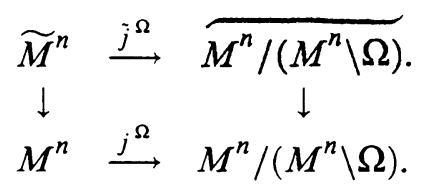

Suppose there is a ball $B\left(p_{0}, r\right)$ such that the boundary of $M^{n} \backslash B\left(p_{0}, r\right)$ has two or more connected components. Then $\pi_{1}\left(M^{n}\right)$ contains an infinite cyclic group by van Kampen's Theorem. Now a contradiction to inequality (4.5) arises, since $j_{*}^{\Omega}$ is injective on this infinite subgroup of $\pi_{1}\left(M^{n}\right)$, provided $\Omega$ is chosen sufficiently large.

\section{REFERENCES}

[A] U. Abresch, Lower curvature bounds, Toponogov's Theorem, and bounded topology, I, II, Ann. Sci. Ecole Norm. Sup. (4) 18 (1985), 651-670 and 20 (1987), 475-502.

[An] M. T. Anderson, On the topology of complete manifolds of nonnegative Ricci curvature, Topology (to appear).

[AW] S. Aloff and N. R. Wallach, An infinite family of distinct 7-manifolds admitting positively curved Riemannian structures, Bull. Amer. Math. Soc. 81 (1975), 93-97.

[BB] L. Bérard Bergery, Quelques exemples des variétés riemanniennes complètes non compactes à courbure de Ricci positive, C. R. Acad. Sci. Paris Sér. I 302 (1986), 159-161.

[BC] R. Bishop and R. Crittenden, Geometry of manifolds, Academic Press, New York, 1964.

[CE] J. Cheeger and D. Ebin, Comparison theorems in Riemannian geometry, North-Holland, New York, 1975.

[CG1] J. Cheeger and D. Gromoll, On the structure of complete manifolds of nonnegative curvature, Ann. of Math. (2) 96 (1972), 413-443.

[CG2] _ The splitting theorem for manifolds of nonnegative Ricci curvature, J. Differential Geom. 6 (1971), 119-128.

[CGT] J. Cheeger, M. Gromov, and M. Taylor, Finite propagation speed, kernel estimates for functions of the Laplace operator, and the geometry of complete Riemannian manifolds, $\mathrm{J}$. Differential Geom. 17 (1982), 15-53.

[EH] J. Eschenburg and E. Heintze, An elementary proof of the Cheeger-Gromoll splitting theorem, Ann. Global Anal. Geom. 2 (1984), 141-151.

[Gal] G. Galloway, A generalization of the Cheeger-Gromoll Splitting Theorem, Arch. Math. 47 (1986), 372-375. 
[GM1] D. Gromoll and W. T. Meyer, On complete manifolds of positive curvature, Ann. of Math. (2) 90 (1969), 75-90.

[GM2] __ Examples of complete manifolds with positive Ricci curvature, J. Differential Geom. 21 (1985), 195-211.

[G] M. Gromov, Curvature, diameter and Betti numbers, Comment. Math. Helv. 56 (1981), 179-195.

[GLP] M. Gromov, J. Lafontaine, and P. Pansu, Structures métriques pour les variétés Riemanniennes, Nathan, Paris, 1982.

[Gr] K. Grove, Metric differential geometry (Nordic Summer School 1985), Lecture Notes in Math., vol. 1263, Springer-Verlag, Berlin and New York, 1987.

[GS] K. Grove and K. Shiohama, A generalized sphere theorem, Ann. of Math. (2) 106 (1977), 201-211.

[L] H. B. Lawson, The unknottedness of minimal embeddings, Invent. Math. 11 (1970), 183187.

[M] J. Milnor, A note on curvature and fundamental group, J. Differential Geom. 2 (1968), 1-7.

[MS] V. D. Milman and G. Schechtman, Asymptotic theory of finite dimensional normed spaces (Appendix: Isoperimetric inequalities in Riemannian manifolds, by M. Gromov), Lecture Notes in Math., vol. 1200, Springer-Verlag, Berlin and New York, 1986.

[MSY] W. Meeks III, L. Simon, and S. T. Yau, Embedded minimal surfaces, exotic spheres, and manifolds with positive Ricci curvature, Ann. of Math. 116 (1982), 621-659.

[ShY] J. P. Sha and D. G. Yang, Examples of manifolds of positive Ricci-curvature, J. Differential Geom. 29 (1989), 95-103.

[ShY1] _ Positive Ricci curvature on the connected sums of $S^{n} \times S^{m}$, J. Differential Geom. (to appear).

[SY] R. Schoen and S. T. Yau, Existence of incompressible minimal surfaces and the topology of three dimensional manifolds with nonnegative scalar curvature, Ann. of Math. 110 (1979), 127-142.

[Y1] S. T. Yau, Seminar on differential geometry, Princeton Univ. Press, Princeton, NJ, 1982.

[Y2] Some function theoretic properties of complete Riemannian manifolds, and their applications to geometry, Indiana Univ. Math. J. 25 (1976), 659-670.

Mathematisches Institut der Universität, Einsteinstr. 64, 4400 Münster, Federal REPUBLIC OF GERMANY

Department of Mathematics, State University of New York at Stony Brook, Stomy BROOK, NEW YORK 11794 